\title{
Multi-Drug Resistant CMV Viremia Secondary to a Rare Viral Mutation in a Transplant Recipient Successfully Treated with Anti-Proliferative Cessation and Immunoglobulins
}

Eleni Papakanaki ${ }^{1}$, Rachel Folwell ${ }^{1}$, Grainne Walsh ${ }^{1}$, Helen E Jones ${ }^{1}$, Eithne MacMahon ${ }^{2}$ and Jelena Stojanovic ${ }^{1}$

1. Department of Paediatric Nephrology, Evelina London Children's Hospital, London, UK

2. Department of Infectious Diseases, Guy's and St Thomas NHS Foundation Trust, London, UK

\section{Introduction}

- Post-transplant viremias are an emerging clinical challenge in paediatric transplant recipients.

-In developed countries, $60 \%$ of children in general population are CMV seronegative at the age of 18 years(1).

- Subclinical CMV viremia is associated with an increased risk of chronic allograft injury(2) and adds to patient morbidity therefore requiring prompt treatment.

- International Transplant Society for CMV Consensus Group advises Ganciclovir, Valganciclovir and Foscarnet as treatment options for CMV viremia(3).

-Viral DNA polymerase resistance mutations are rare but may be associated with cross resistance adding to the challenges when treating such patients.

- We report a rare $\mathrm{CMV}$ mutation associated with resistance to all available medications in a CMV seronegative recipient successfully treated with reduction in immunosuppression and intravenous immunoglobulins (IVIG).

\section{Case Report}

-A 16 year-old girl with ESRD due to Autosomal Recessive Alport Syndrome.

-She was on renal replacement therapy for three years.

- She received a deceased donor kidney transplant (mismatch 1-2-0, CMV IgG Donor positive / Recipient negative; EBV Donor negative/ Recipient positive).

\section{Immunosuppression:}

Induction: Intravenous Basiliximab 20mg on days 0 and 4.

Maintenance: Tacrolimus, Azathioprine and Prednisolone.

CMV Prophylaxis: Valganciclovir for 90 days post transplant (our centre's protocol).

\section{Post transplant course:}

Day 44: CMV DNA was first detected with a CMV load of $4964 \mathrm{IU} / \mathrm{mL}$ (CMV load log value 3.97). She remained asymptomatic with no evidence of clinical CMV disease.

Day 51: prophylaxis changed to treatment dose oral Valganciclovir (Arrow 1, Graph 1).

Day 59: reduction in Azathioprine dose (Arrow 2, Graph 1).

Day 72: A 5-day period of non-compliance with antiviral treatment corresponded with further increase in CMV load log (Arrow 3, Graph 1).

Day 110: a 7-day course of intravenous Ganciclovir given in view of persistently high CMV viral loads. Despite this, the viral load increased to a maximum log value of 5.41 .

A rare UL54 deletion mutation (POL gene deletion 981/982) was detected conferring high level resistance to Ganciclovir and low level resistance to Foscarnet and Cidofovir.

Day 123: IVIG given (dose 1g/kg), Azathioprine stopped (Arrow 4, Graph 1) and Prednisolone changed from alternate days to daily regime. This was associated with a reduction in CMV log count from 4.41 to 4.18 .

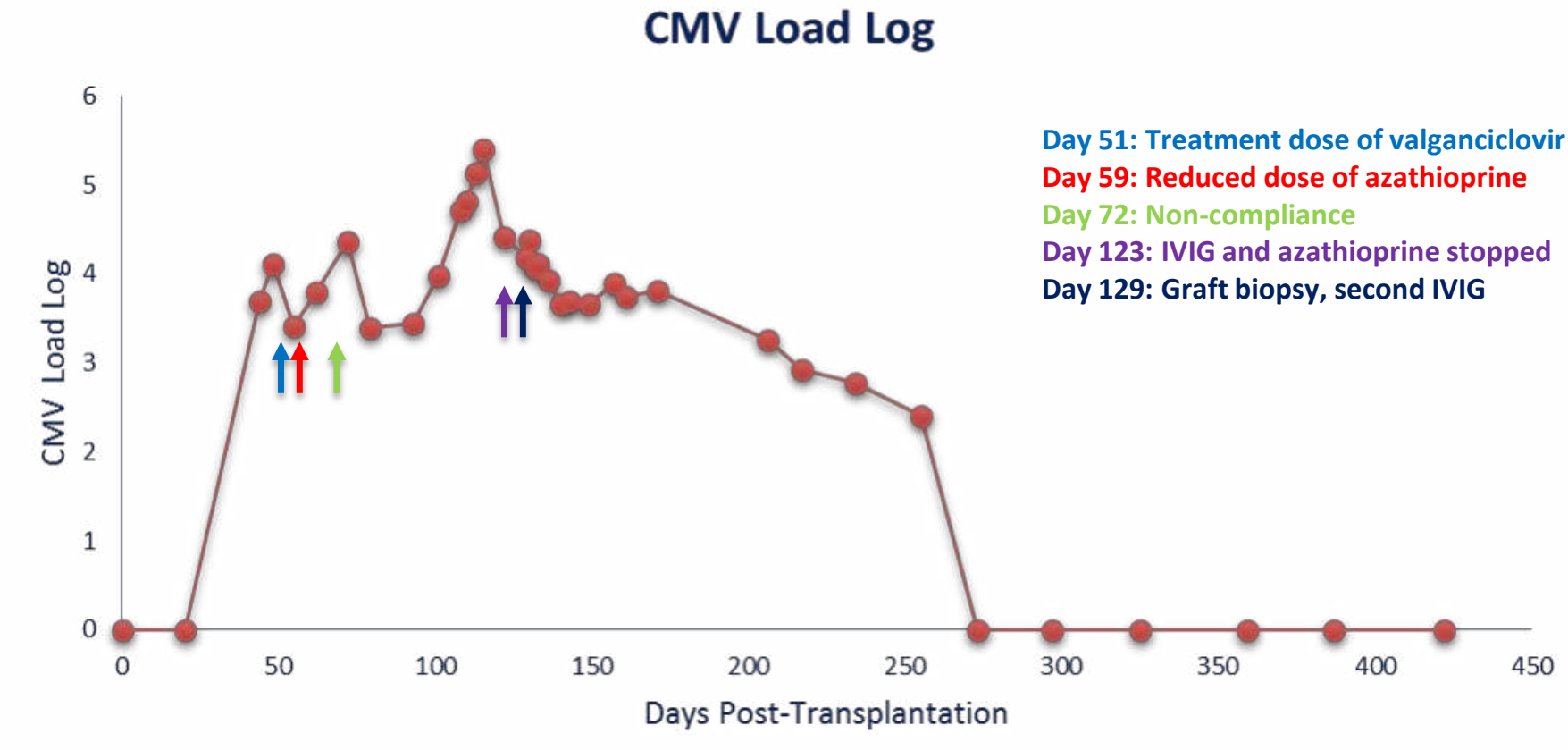

Graph 1 :CMV Viral Load from transplant until 16 months post transplant

Creatinine Level (umol/L)

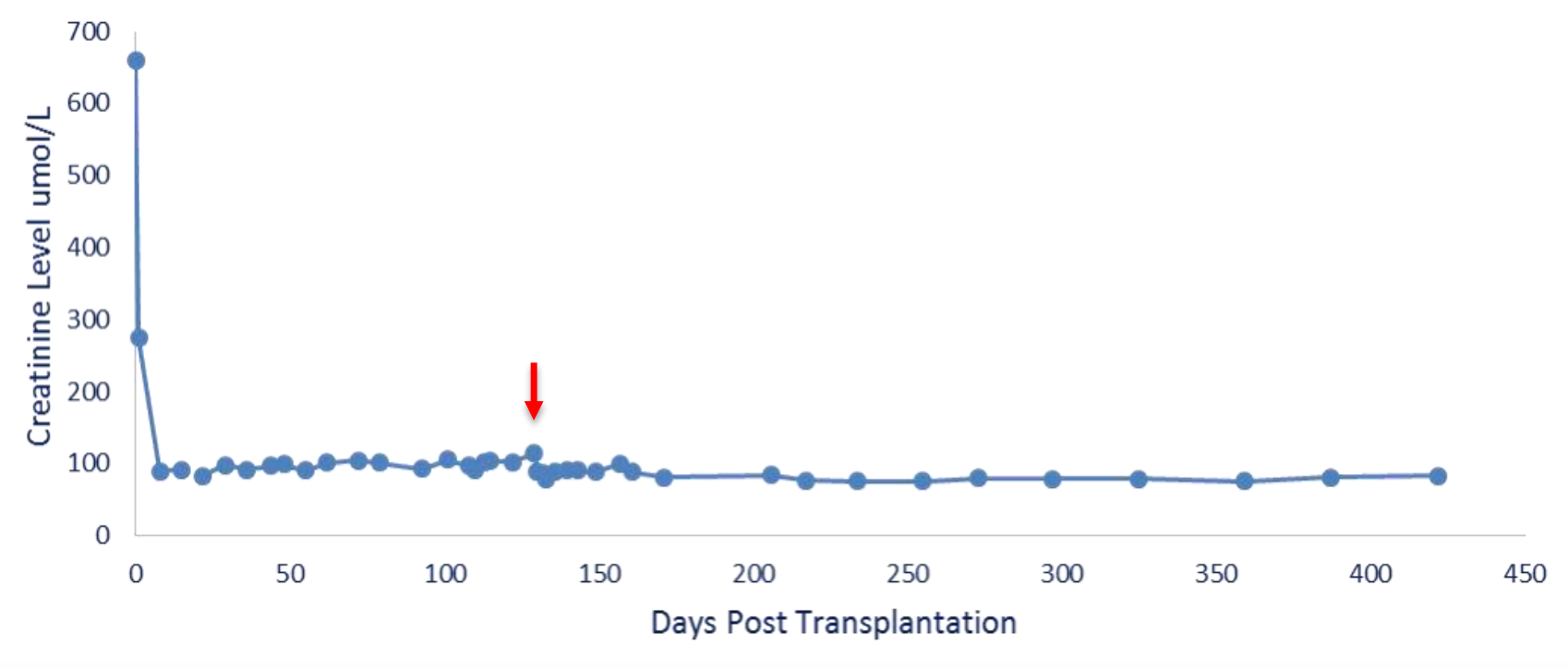

Graph 2: Stable graft function measured by serum creatinine with a rise (Arrow) associated with borderline T cell mediated rejection

Day 129: Rise in creatinine (from a baseline of $85 \mathrm{umol} / \mathrm{L}$ to $147 \mathrm{umol} / \mathrm{L}$ ) coinciding with a 5-day history of non-adherence with daily Prednisolone (Graph 2).

Graft biopsy confirmed borderline T-cell mediated rejection and CNI toxicity (Banff criteria 3), with no evidence of CMV inclusions and negative immunoperoxidase staining. Treatment included a second dose of IVIG $(1 \mathrm{~g} / \mathrm{kg})$ and two doses of high dose oral Prednisolone (Arrow 5, Graph 1). Creatinine improved.

Serial CMV load levels demonstrated a stable viremia (Graph 1).

Day 273: CMV DNA first undetectable.

Sixteen months: Patient remains well, has a stable graft function (eGFR $69 \mathrm{ml} / \mathrm{min} / 1.73 \mathrm{~m} 2$ ) and continues on dual immunosuppression with $\mathrm{CNI}$ and a low-dose daily Prednisolone only. CMV DNA remains undetectable. DSA negative.

\section{Discussion}

We present a case of subclinical multi-resistant CMV viremia successfully treated with a reduction in immunosuppression and intravenous immunoglobulins with good patient and graft outcome.

Although rare, viral mutations conferring multi-drug resistance present an emerging therapeutic challenge. The successful outcome in this patient provides a possible treatment framework for other clinicians. This might be particularly useful in countries where access to anti viral medication is difficult.

\section{References}

